\title{
Effect of Bedrock Stiffness and Thickness of Weathered Rock on Response Spectrum in Korea
}

\author{
Satish Manandhar*, Hyung Ik Cho**, and Dong Soo Kim*** \\ Erratum to: KSCE Journal of Civil Engineering (2016) 20(7):2677-2691 \\ DOI 10.1007/s12205-016-0811-z
}

The original version of this article unfortunately contained a mistake.

Errata:

1. The order of the first and last name of the below author was written incorrectly.

Manandhar Satish

Will be corrected as

Satish Manandhar.

*Researcher, Dept. of Civil and Environmental Engineering, KAIST, Daejeon 34141, Korea (E-mail: satish@kaist.ac.kr)

**Graduate Student, Dept. of Civil and Environmental Engineering, KAIST, Daejeon 34141, Korea (E-mail: goodchoik@kaist.ac.kr)

***Member, Professor, Dept. of Civil and Environmental Engineering, KAIST, Daejeon 34141, Korea (Corresponding Author, E-mail: dskim@kaist.ac.kr) 